\title{
FUNCIONAMIENTO EJECUTIVO EN EL TRASTORNO DE DÉFICIT DE ATENCIÓN CON HIPERACTIVIDAD: UNA PERSPECTIVA ECOLÓGICA DE LOS PERFILES DIFERENCIALES ENTRE LOS TIPOS COMBINADO E INATENTO
}

\author{
M. INMACULADA NAVARRO ${ }^{1}$ y DOMINGO A. GARCÍA-VILLAMISAR ${ }^{2}$ \\ ${ }^{1}$ Colegio Público Giner de los Ríos, Albacete, España \\ ${ }^{2}$ Facultad de Educación, Universidad Complutense de Madrid, Madrid, España
}

\begin{abstract}
Resumen: Este estudio trata de definir las disfunciones ejecutivas implicadas en el trastorno de déficit de atención con hiperactividad (TDAH) y, concretamente, en los tipos inatento y combinado. Además, pretende determinar si son los síntomas de inatención o los de hiperactividad/impulsividad de este trastorno los que están relacionados con dichas disfunciones. Mediante la escala Behavior Rating Inventory of Executive Function (BRIEF) se evaluaron 87 niños y se clasificaron en tres grupos: 29 con TDAH inatento, 29 con TDAH combinado, y 29 sin TDAH (grupo de control). Los resultados muestran que los niños con TDAH se diferencian del grupo de control en todas las dimensiones de la escala BRIEF. Las diferencias entre los tipos de TDAH estuvieron centradas en los aspectos comportamentales más que en los cognitivos, siendo el tipo combinado el más afectado. Además, son los síntomas de inatención los que están relacionados, en mayor medida, con la escala BRIEF.
\end{abstract}

Palabras clave: Funciones ejecutivas; hiperactividad; impulsividad; inatención; TDAH.

Executive functioning in attention deficit hyperactivity disorder (ADHD): An ecological perspective of the differential profiles between the combined and the inattentive types

\begin{abstract}
This study aims to define the executive dysfunctions in attention deficit hyperactivity disorder (ADHD) and, specifically, in the inattentive and combined subtypes. Moreover, it intends to determine whether either the symptoms of inattention or those of hyperactivity/impulsivity of the disorder are related to these dysfunctions. Using the BRIEF (Behavior Rating Inventory of Executive Function), a total of 87 children were assessed and classified in three groups: 29 children with inattentive ADHD, 29 children with combined ADHD, and a control group of 29 children without ADHD. The results show that the children with ADHD differ from the control group in all dimensions of the BRIEF. The differences between ADHD types were more marked in behavioral than in cognitive aspects, the combined type being most affected. Furthermore, inattention symptoms appeared to be more specifically related to the BRIEF.
\end{abstract}

Keywords: Executive functions; hyperactivity; impulsivity; inattention; ADHD.

\section{INTRODUCCIÓN}

El trastorno de déficit de atención con hiperactividad (TDAH) es uno de los trastornos de la infancia más comúnmente diagnosticados, con índices de prevalencia entre un 3 y un 7\%

Recibido: 23 septiembre 2010; aceptado 12 diciembre 2010

Correspondencia: Dra. M. Inmaculada Navarro González, Colegio Público Giner de los Ríos, Doctor Galiacho 4, $3^{\circ}$ I, 02006 Albacete (España). Correo-e: navar@ono.com
(American Psychiatric Association; APA, 2000). El TDAH se caracteriza por unos niveles de inatención, hiperactividad e impulsividad inapropiados desde el punto de vista evolutivo (Fonseca-Pedrero, Paino, Lemos-Giráldez y Muñiz, 2011). Estos síntomas se inician en la primera infancia, son de naturaleza relativamente crónica y no pueden atribuirse a alteraciones neurológicas, sensoriales, del lenguaje o motoras graves, a discapacidad intelectual o a tras- 
tornos emocionales severos (Barkley, 2006; Frías, Carrasco, Fernández, García y García, 2009). En un amplio porcentaje de casos, este trastorno se manifiesta con un notable déficit en la capacidad de inhibición que interfiere en el rendimiento efectivo de las funciones ejecutivas. Estas funciones ejecutivas a las que nos referimos se suelen definir, en términos globales, como un amplio abanico de operaciones cognitivas que pueden guardar entre sí una relación lejana (Ozonoff, 2000); entre ellas, se incluyen las habilidades vinculadas a la capacidad de organizar y planificar una tarea, seleccionar apropiadamente los objetivos, iniciar un plan y sostenerlo en la mente mientras se ejecuta, inhibir las distracciones, cambiar de estrategia, en caso necesario, de modo flexible; autorregular y controlar el curso de la acción para asegurarse de que el objetivo propuesto está en vías de conseguirse (DeBonis, Ylvisaker y Kundert, 2000). Las funciones ejecutivas comprenden al menos cuatro factores: (a) inhibición de respuesta y ejecución, (b) memoria de trabajo $\mathrm{y}$ actualización de datos, (c) cambio de tarea y (d) control de la interferencia (Barkley, Edwards, Laneri, Fletcher y Metevia, 2001; Miyake, Friedman, Emerson, Witzki y Howerter, 2000; Pennington y Ozonoff, 1996; Robbins et al., 1998; Sergeant, Geurts, Huijbregts, Scheres y Oosterlaan, 2003; Willcutt, Chhabildas y Pennington, 2001; Willcutt, Pennington, Chhabildas, Olson, Hulslander, 2005). Según Barkley (1997), a medida que el individuo alcanza la madurez, las funciones ejecutivas se van interiorizando y se convierten en privadas. A juicio de este y otros autores, el proceso de internalización no se completa en el caso de los niños con TDAH (Barkley, 1997; Barkley, Grodzinsky y DuPaul, 1992; Berlin, Bohlin, Nyberg y Janols, 2004; Hinshaw, Carte, Fan, Jassy y Owens, 2007; Weyandt, Rice, Linterman, Mitzlaff y Emert, 1998). Otros investigadores, no suscriben esta asociación entre disfunción ejecutiva y TDAH (Geurts, Verté, Oosterlaan, Roeyers y Sergeant, 2004; Scheres et al., 2004) y se muestran partidarios de no atribuir un déficit generalizado en el funcionamiento ejecutivo en los niños afectados por este trastorno.

Con respecto a los tipos del TDAH (inatento, combinado e hiperactivo/impulsivo), ciertos estudios constatan que sólo el tipo combinado presenta algún déficit específico en el funcionamiento ejecutivo (Barkley, 1997; Houghton et al., 1999; Nigg, Blaskey, Huang-Pollock y Rappley, 2002); otros, sin embargo, no aprecian diferencia alguna entre los tipos combinado e inatento (Chhabildas, Pennington y Willcutt, 2001; Hinshaw et al., 2007; Sheres et al., 2004;). Nos parece conveniente aclarar que son pocos los estudios que han incluido al tipo hiperactivo/ impulsivo (Chhabildas et al., 2001). Según Nigg (2006) este tipo es menos válido etiológicamente, es decir, tiene menos solidez con vistas a la validez del trastorno que los otros dos tipos. Tradicionalmente, se han encontrado mayor número de déficits en el tipo combinado que en el inatento, lo cual, según Nigg (2006), parece mostrar que, en un significativo número de casos, el TDAH inatento puede ser una variante de la condición vista en el tipo combinado.

El funcionamiento ejecutivo, visto desde una perspectiva ecológica, que planteamos en esta investigación, ayuda, en parte, a solventar la baja correspondencia del funcionamiento ejecutivo con los procesos comportamentales que algunos autores (Hughes y Graham, 2002) aluden en referencia a la problemática en la evaluación de las funciones ejecutivas. Nuestro trabajo, que se aplica a nivel escolar, estudia las situaciones en el centro educativo y su entorno y el cómo responden los niños a ellas (Shulman, 1986) avanzando así en la aplicación de programas de tratamiento contextualizados y personalizados (Ferro, Vives y Ascanio, 2010; Pelechano, 2007).

Los objetivos de esta investigación son: (a) evaluar las posibles alteraciones que los grupos con TDAH, frente al grupo de control, manifiestan en el funcionamiento ejecutivo, (b) determinar si los grupos con TDAH de tipo inatento y con TDAH de tipo combinado presentan o no diferentes alteraciones en el funcionamiento ejecutivo, (c) precisar en qué medida la sintomatología inatencional o la de hiperactividad/impulsividad están relacionadas con la capacidad de metacognición y el control del comportamiento incluidos en los procesos ejecutivos y (d) contribuir al establecimiento de un perfil ejecutivo particular que pueda distin- 
guir ambos tipos de TDAH y que constituya la base para el establecimiento de un tratamiento eficaz.

El planteamiento de estos objetivos nos 1leva a establecer las siguientes hipótesis: (a) se espera que los grupos con TDAH, con respecto al grupo de control, evidencien alteraciones en las variables ecológicas sobre funcionamiento ejecutivo, (b) se espera que el grupo con TDAH de tipo inatento muestre alteraciones en la capacidad de metacognición del funcionamiento ejecutivo y que el grupo con TDAH de tipo combinado muestre alteraciones tanto en la capacidad de metacognición como en el control comportamental de dicho funcionamiento ejecutivo y (c) se espera que las variables ecológicas que evalúan las disfunciones en la capacidad de metacognición estén relacionadas con la sintomatología de inatención y que aquellas que evalúan las disfunciones en el control del comportamiento estén relacionadas con la sintomatología de hiperactividadimpulsividad.

\section{MÉTODO}

\section{Participantes}

La población de partida para la realización de este estudio fue de 789 niños escolarizados entre $3^{\circ}$ y $6^{\circ}$ de Educación Primaria, elegidos al azar de entre 6 centros públicos y 2 centros concertados seleccionados aleatoriamente en la capital y provincia de Albacete. La muestra definitiva quedó constituida por 87 niños repartidos en tres grupos: (a) niños con TDAH de tipo inatento (TDAH/TI) $(n=29)(65,5 \%$ de niños y $34,5 \%$ de niñas), (b) niños con TDAH de tipo combinado (TDAH/TC) $(n=29)(86,2 \%$ de niños y $13,8 \%$ de niñas)y (c) niños sin TDAH como grupo de control (Control) $(n=29)$ (62,1\% de niños y $37,9 \%$ de niñas). Todos ellos eran hispano-hablantes y asistían a la escuela con regularidad. La edad de los niños de la muestra oscila entre los 9 y los 13 años; en el grupo de control la media es de 10,93 años (DT $=1,25)$, en el grupo con TDAH de tipo inatento es de $11,24(D T=1,15)$ y en el grupo con el tipo combinado es de11,03 $(D T=1,35)$. El rendimiento académico del grupo de control fue superior que el de los grupos con TDAH $\left(\chi_{(3,87)}^{2}\right.$ $=64,11, p=0,00)$. En el grupo de control se incluyó a aquellos niños con ausencia de sintomatología de TDAH en la escala de Conners (Conners, 1969) así como en los ítems del Manual Diagnóstico y Estadístico de los Trastornos Mentales (DSM-IV-TR; APA, 2000). Ninguno de los niños incluidos en el estudio presentaba defectos visuales o auditivos, enfermedad médica (cáncer, diabetes), epilepsia, discapacidad intelectual, trastornos generalizados del desarrollo (tipo autismo), trastornos emocionales o problemas sociales. Todos los profesores-tutores y padres o tutores dieron el correspondiente consentimiento informado.

\section{Instrumentos de evaluación}

Cuestionario de Conducta de Conners para profesores en su versión abreviada (ASQ: Abbreviated Sympton Questionnaire, Conners, 1969). El cuestionario consta de 10 ítems que los profesores-tutores valoraron de acuerdo a los siguientes criterios: Nada (0 puntos), Poco (1 punto), Bastante ( 2 puntos) y Mucho (3 puntos) según el grado en que el niño manifestase la conducta expresada en cada ítem. La valoración máxima es de 30 puntos y la mínima de 0 puntos. La mayor parte de los autores consideran que el Cuestionario de Conducta de Conners es adecuado para valorar la sintomatología del TDAH (Brito, 1987; Conners, Sitarenios, Parker y Epstein, 1998; Crystal, Ostrander, Chen y August, 2001; Goyette, Conners y Ulrich, 1978; Kazdin, Esveldt-Dawson y Loar, 1983; Maganto, 1995; Rowe y Rowe, 1997; Valero, 1997). Aunque existe una escala Conners revisada, no estaba adaptada al español en el momento de realizar esta investigación (ver Conners et al., 1998).

Evaluación según criterios del DSM-IV-TR (APA, 2000). Con el fin de valorar a los participantes según los criterios clínicos del DSMIV-TR (APA, 2000), se elaboró un cuestionario tipo Likert con una escala de valoración de 0 a 3 puntos que contenía los síntomas específicos de cada uno de los tipos establecidos según dichos criterios. El cuestionario está adaptado 
de acuerdo al procedimiento diseñado en otras investigaciones (Barkley y Murphy, 1998; Chhabildas et al., 2001; DuPaul et al., 1998; Lahey et al., 1998) y consta de 18 ítems, 9 de ellos pertenecientes a la dimensión inatención y 9 a la dimensión hiperactividad-impulsividad. Los ítems de ambas dimensiones fueron alternados de forma aleatoria para evitar el efecto halo (Martínez Arias, 1995). Las posibilidades de respuesta para cada ítem fueron: Nada (0 puntos), Un Poco (1 punto), Bastante (2 puntos) y Mucho (3 puntos) según el grado en el que el niño manifestara la conducta propuesta.

Behavior Rating Inventory of Executive Function (BRIEF; Gioia, Isquith, Guy y Kenworthy, 2000) para la evaluación del funcionamiento ejecutivo. Es una escala compuesta por dos cuestionarios de 86 ítems, uno para padres y otro para profesores, diseñados para evaluar el funcionamiento ejecutivo en el hogar y en la escuela, respectivamente. Determina en qué medida las disfunciones ejecutivas se reflejan en la vida real, es decir, la validez ecológica. La escala tiene unas sólidas propiedades psicométricas (p.ej., la fiabilidad test-retest es de $r=$ $0,82)$ y la validez convergente ha sido establecida con otras medidas de inatención, impulsividad y de aprendizaje (Gioia et al., 2000). En esta investigación hemos utilizado la escala BRIEF para profesores que, como dijimos, estos han cumplimentado para cada uno de los niños incluidos en el estudio. Una mayor puntuación en esta escala es señal de un mayor número de alteraciones en las funciones ejecutivas. Cada ítem tiene tres opciones de respuesta: Nunca (1 punto), A veces ( 2 puntos), Con frecuencia (3 puntos), según la asiduidad con la que el niño realice las conductas expresadas en cada cuestión. Los ítems se agrupan en 8 dimensiones, a saber: (a) Inhibición, (b) Cambio, (c) Control Emocional, (d) Iniciativa, (e) Memoria de Trabajo, (f) Planificación-Organización, (g) Organización de Materiales y (h) Control. A continuación presentamos una breve descripción de cada una de ellas:

Inhibición: Habilidad para resistirse a los impulsos y detener la conducta en el momento adecuado. Cambio: Habilidad para hacer y tolerar cambios, flexibilidad para resolver pro- blemas y pasar el foco atencional de un tema a otro cuando se requiere. Control Emocional: Influencia de las funciones ejecutivas en la expresión y regulación de las emociones. Iniciativa: Habilidad para iniciar una actividad sin ser incitado a ello. Memoria de Trabajo: Capacidad para mantener la información en la mente durante un breve periodo de tiempo con el objetivo de completar una tarea, registrar y almacenar información o generar objetivos. Organización y Planificación: Estos componentes son necesarios para la resolución de problemas. La organización implica la habilidad para identificar las ideas principales o conceptos clave de una información. La planificación supone el planteamiento de un objetivo y la elección de la mejor vía para alcanzarlo, con frecuencia, a través de pasos adecuadamente secuenciados. Organización de Materiales: Es otro aspecto de la organización que refleja la habilidad para ordenar elementos del entorno. Control: Esta dimensión comprende dos aspectos; el primero, se refiere al control del propio rendimiento durante la realización de una tarea o al finalizar la misma para cerciorarse de que se ha alcanzado el objetivo. El segundo aspecto, refleja la conciencia del niño acerca de los efectos que su conducta provoca en los demás.

Las dimensiones se agrupan en dos índices principales: (a) Índice de Regulación Comportamental (IRC) que incluye las dimensiones de Inhibición, Cambio y Control Emocional y (b) Índice de Metacognición (IM) que agrupa las dimensiones de Iniciativa, Memoria de Trabajo, Planificación-Organización, Organización de Materiales y Control. Estos dos índices se agrupan en la llamada Composición Ejecutiva Global (CEG). A las 8 dimensiones mencionadas se añaden 13 ítems adicionales, estos ítems no se miden y, por tanto, no contribuyen a la puntuación. Su existencia se justifica por la utilidad para establecer una intervención funcional y por su relevancia en el estudio de las poblaciones clínicas específicas como, por ejemplo, en el caso del TDAH. Estos ítems fueron originalmente asignados a las dimensiones de Inhibición, Planificación-Organización, Control, Cambio y Memoria de Trabajo. 
Isquith y Gioia (2000) examinaron mediante regresión logística 2 de las 8 dimensiones de este cuestionario, concretamente las de Memoria de Trabajo y de Inhibición. El objetivo del estudio fue determinar su sensibilidad y especificidad a la hora de identificar sujetos con y sin TDAH y sus tipos, en niños referidos clínicamente. Las escalas de padres, en la dimensión de Memoria de Trabajo, identificaron al 89\% de los sujetos del tipo inatento y al $85 \%$ del tipo combinado con medidas $\mathrm{T}$ en o por encima del percentil 90, mientras que identificaron de forma incorrecta al $16 \%$ de los niños del grupo de control. Una puntuación de corte en el percentil 90 para la escala de Inhibición identificó correctamente al $85 \%$ de los niños con diagnóstico del tipo combinado e identificó incorrectamente al 14\% sin este trastorno. Las escalas de maestros fueron similares en lo que a sensibilidad de refiere, con una puntuación en la escala de Memoria de Trabajo en el percentil 90 identificando al 76\% de los niños con el tipo inatento pero sólo al $17 \%$ de los niños sin un diagnóstico de TDAH. Una puntuación en o por encima del percentil 88 en la escala de Inhibición identificó al $78 \%$ de los niños con el tipo combinado y al $21 \%$ de los controles.

Además de esta utilidad diagnóstica, la escala BRIEF puede delimitar la amplitud de las áreas potenciales de la disfunción ejecutiva en el TDAH (p.ej. organización, planificación, déficits de control, descontrol emocional) así como definir funcionalmente diversos aspectos de la disfunción ejecutiva, lo cual puede añadir información válida para la evaluación del TDAH.

\section{Procedimiento}

El procedimiento aplicado en esta investigación siguió una metodología propia de los estudios de doble fase; a saber, una primera fase de screening y una segunda fase de selección de los sujetos de la muestra que participaron en el estudio. La selección de los sujetos en la fase de screening se realizó mediante el Cuestionario de Conducta de Conners para profesores en su versión abreviada. El objetivo de la aplicación de este cuestionario es la identificación de sujetos en los que se puede sospechar la presencia de sintomatología propia del TDAH. Una identificación positiva de este tipo de síntomas (puntuación superior a 17 para los niños y mayor de 12 para las niñas) (Orjales Villar, 2002) dio paso a la segunda fase mediante los ítems del DSMIV-TR para confirmar o descartar la presencia de sintomatología de TDAH.

En esta segunda fase se identificaron los grupos con sintomatología de TDAH, de tipo inatento y de tipo combinado, y el grupo de control. Para el grupo de control fueron seleccionados de forma aleatoria 29 niños con una puntuación igual o menor a 4 en el Cuestionario Abreviado de Conners. Se descartaron aquellos sujetos que puntuaron de forma positiva (Bastante o Mucho) en el ítem 3 de dicho cuestionario ("Se distrae fácilmente, tiene escasa atención") con el fin de evitar la selección de sujetos que pudieran manifestar algún tipo de sintomatología inatencional. Los grupos con sintomatología de TDAH de tipo inatento y de tipo combinado se establecieron a través de la aplicación de los criterios del DSM-IV-TR (APA, 2000). Como ha sido sugerido por otros investigadores (p.ej. Chhabildas et al., 2001; Hartman, Willcutt, Rhee y Pennington, 2004) los ítems del DSM-IV-TR señalados con 2 y 3 puntos fueron considerados como signos positivos de TDAH. El máximo de signos positivos que se pueden obtener así es de 9 en la dimensión inatención (IA) y 9 en la dimensión hiperactividad-impulsividad (H/I). Aquellos niños que mostraron 6 ó más síntomas de IA pero menos de 6 síntomas de H/I fueron seleccionados para el grupo con TDAH de tipo inatento. Los niños que mostraron 6 ó más síntomas de H/I y 6 ó más síntomas de IA fueron seleccionados para el grupo con TDAH de tipo combinado. El cuestionario con los criterios del DSMIV-TR fue cumplimentado por cada uno de los profesores-tutores, posteriormente entrevistamos a cada uno de dichos profesores en relación a los diversos síntomas del TDAH incluidos. La selección de los sujetos con este procedimiento no supone un diagnóstico clínico sino sólo la identificación de casos con sintomatología propia del TDAH, lo cual reconocemos como una importante limitación del estudio.

La fiabilidad en el uso de los criterios del DSM-IV-TR para el TDAH viene avalada por 
diversas investigaciones. En el estudio de Barkley et al. (2001) la consistencia interna (coeficiente alpha) fue de 0,92 y la fiabilidad testretest con un intervalo de tiempo de 1 mes fue de 0,85. Gomez, Harvey, Quick, Scharer y Harris (1999) expusieron un valor de consistencia interna en las escalas de padres de 0,92 en los ítems de inatención y de 0,90 en los de hiperactividad-impulsividad. En cuanto a las escalas de profesores, Gomez et al. (1999) ofrecieron una consistencia interna de 0,95 en las escalas de inatención y de 0,94 en las de hiperactividadimpulsividad. Según estos mismos autores, la fiabilidad test-retest al cabo de 3 meses fue de 0,55 en ambas escalas de padres (ítems de inatención y de hiperactividad-impulsividad). Esta fiabilidad fue, en las escalas de profesores, de 0,70 para los ítems de inatención y de 0,73 para los de hiperactividad-impulsividad.

En líneas generales, la validez convergente ha sido establecida a través de correlaciones entre los ítems sobre el TDAH del DSM-IV-TR y los de otras escalas que evalúan el comportamiento hiperactivo-impulsivo con un índice entre 0,61 y 0,80 para las escalas de padres (Barkley et al., 2001). Los síntomas de inatención y de hiperactividad-impulsividad del DSM-IV mostraron una validez superior a 0,75 con la escala abreviada de Conners (Gomez, Burns, Walsh y Alves de Moura, 2003).

\section{Análisis estadístico}

El proceso de análisis de divide en dos etapas. La primera de ellas se explica desde una perspectiva categorial estableciendo comparaciones entre los tipos del TDAH (según el DSM-IV-TR) y el grupo de control con respecto al funcionamiento ejecutivo. La segunda etapa se desarrolla desde un punto de vista dimensional, en ella hemos realizado un análisis correlacional para determinar si existe o no relación entre la sintomatología del TDAH y las variables ecológicas sobre funcionamiento ejecutivo (puntuación en la escala BRIEF). En la primera etapa, de análisis categorial, utilizamos un análisis de varianza con la variable edad como covariante (Chhabildas et al., 2001; Hoza et al., 2004; Hoza, Pelham, Waschbusch, Kipp y Owens, 2001; Nigg, Blaskey, Stawicki y Sachek, 2004; Nigg et al., 2005) para comparar los grupos con TDAH y el grupo de control en cuanto a la puntuación en todas las dimensiones de la escala BRIEF. La inclusión de esta covariante se justifica porque estimamos que una variación de 4 años (la edad de los niños en todos los grupos oscila entre los 9 y los 13 años) podía influir en la puntuación obtenida en la escala BRIEF. Para precisar las diferencias existentes empleamos los tests post-hoc de Tuckey y Games-Howell (Games, Keselman y Rogan, 1981) según pudimos asumir o no la igualdad de varianzas (prueba de Levene).

En la segunda etapa, desde una perspectiva dimensional, realizamos un análisis correlacional parcial, con la variable edad como variable controlada para, como dijimos, establecer si hay o no relación entre la sintomatología del TDAH y la capacidad de metacognición y el control del comportamiento del funcionamiento ejecutivo (puntuación en la escala BRIEF).

\section{RESULTADOS}

En los párrafos que siguen mostramos los resultados obtenidos en relación a cada una de las hipótesis planteadas. De acuerdo con la primera hipótesis (los grupos con TDAH manifestarán, frente al grupo de control, alteraciones en las variables ecológicas que evalúan el funcionamiento ejecutivo) todos las dimensiones de la escala BRIEF (variables ecológicas) mostraron diferencias significativas entre los grupos con TDAH y el grupo de control (véase la Tabla 1). Las puntuaciones siempre fueron mayores en los grupos con TDAH que en el grupo de control. Por ello, suponemos disfunciones ejecutivas en los niños con TDAH ya que la puntuación en este test es inversamente proporcional a la capacidad ejecutiva. La medida de asociación empleada (eta cuadrado) evidenció valores entre 0,55 y 0,90 de ahí que podamos concluir que la variación total en la puntuación de las dimensiones de la escala BRIEF se debió en un alto porcentaje (entre el 55\% y el 90\%) a la pertenencia a un determinado grupo (control, TDAH con tipo inatento o TDAH con tipo combinado). 
Tabla 1. Índices de la escala BRIEF. Medias y desviaciones típicas (entre paréntesis), análisis de varianza y análisis post-hoc. Variable edad como covariante

\begin{tabular}{|c|c|c|c|c|c|}
\hline $\begin{array}{l}\text { Índices } \\
\text { BRIEF }\end{array}$ & $\begin{array}{l}\text { Control } \\
(n=29)\end{array}$ & $\begin{array}{l}\text { TDAH/TI } \\
(n=29)\end{array}$ & $\begin{array}{c}\mathrm{TDAH} / \mathrm{TC} \\
(n=29)\end{array}$ & $\mathrm{F}(2,80)^{*}$ & $\begin{array}{l}\text { Análisis } \\
\text { Post-hoc }^{\mathrm{a}}\end{array}$ \\
\hline Cambio & $12,96(2,84)$ & $19,45(4,90)$ & $20,10(5,41)$ & 19,96 & $\begin{array}{l}\mathrm{C}<\mathrm{TDAH} / \mathrm{TI} \\
\mathrm{C}<\mathrm{TDAH} / \mathrm{TC}\end{array}$ \\
\hline Inhibición & $11,48(2,18)$ & $18,45(4,95)$ & $25,00(3,89)$ & 79,15 & $\begin{array}{l}\mathrm{C}<\mathrm{TDAH} / \mathrm{TI} \\
\mathrm{C}<\mathrm{TDAH} / \mathrm{TC} \\
\mathrm{TDAH} / \mathrm{TI}<\mathrm{TDAH} / \mathrm{TC}\end{array}$ \\
\hline Control emocional & $10,68(2,12)$ & $15,90(4,62)$ & $19,90(5,62)$ & 31,24 & $\begin{array}{l}\mathrm{C}<\mathrm{TDAH} / \mathrm{TI} \\
\mathrm{C}<\mathrm{TDAH} / \mathrm{TC} \\
\mathrm{TDAH} / \mathrm{TI}<\mathrm{TDAH} / \mathrm{TC}\end{array}$ \\
\hline IRC & $35,12(6,23)$ & $53,79(12,61)$ & $65,00(13,01)$ & 49,66 & $\begin{array}{l}\mathrm{C}<\mathrm{TDAH} / \mathrm{TI} \\
\mathrm{C}<\mathrm{TDAH} / \mathrm{TC} \\
\mathrm{TDAH} / \mathrm{TI}<\mathrm{TDAH} / \mathrm{TC}\end{array}$ \\
\hline Iniciativa & $9,16(2,43)$ & $17,21(3,02)$ & $16,90(3,26)$ & 61,83 & $\begin{array}{l}\mathrm{C}<\mathrm{TDAH} / \mathrm{TI} \\
\mathrm{C}<\mathrm{TDAH} / \mathrm{TC}\end{array}$ \\
\hline Memoria de trabajo & $12,36(3,58)$ & $24,69(4,03)$ & $24,97(4,48)$ & 79,26 & $\begin{array}{l}\mathrm{C}<\mathrm{TDAH} / \mathrm{TI} \\
\mathrm{C}<\mathrm{TDAH} / \mathrm{TC}\end{array}$ \\
\hline Planificación-organización & $13,00(4,28)$ & $24,31(3,97)$ & $24,97(5,90)$ & 50,95 & $\begin{array}{l}\mathrm{C}<\mathrm{TDAH} / \mathrm{TI} \\
\mathrm{C}<\mathrm{TDAH} / \mathrm{TC}\end{array}$ \\
\hline Org. de materiales & $8,28(2,85)$ & $13,59(4,42)$ & $15,93(4,00)$ & 27,31 & $\begin{array}{l}\mathrm{C}<\mathrm{TDAH} / \mathrm{TI} \\
\mathrm{C}<\mathrm{TDAH} / \mathrm{TC}\end{array}$ \\
\hline Control & $12,36(3,33)$ & $21,38(4,36)$ & $24,79(4,28)$ & 65,98 & $\begin{array}{l}\mathrm{C}<\mathrm{TDAH} / \mathrm{TI} \\
\mathrm{C}<\mathrm{TDAH} / \mathrm{TC} \\
\mathrm{TDAH} / \mathrm{TI}<\mathrm{TDAH} / \mathrm{TC}\end{array}$ \\
\hline IM & $55,16(15,08)$ & $101,17(16,40)$ & $107,55(18,07)$ & 75,83 & $\begin{array}{l}\mathrm{C}<\mathrm{TDAH} / \mathrm{TI} \\
\mathrm{C}<\mathrm{TDAH} / \mathrm{TC}\end{array}$ \\
\hline CEG & $90,28(20,00)$ & $154,97(24,14)$ & $172,55(28,284)$ & 81,05 & $\begin{array}{l}\mathrm{C}<\mathrm{TDAH} / \mathrm{TI} \\
\mathrm{C}<\mathrm{TDAH} / \mathrm{TC} \\
\mathrm{TDAH} / \mathrm{TI}<\mathrm{TDAH} / \mathrm{TC}\end{array}$ \\
\hline
\end{tabular}

Nota. Las desviaciones típicas van entre paréntesis. Una menor puntuación en la escala BRIEF implica un mejor funcionamiento ejecutivo. TDAH/TI= TDAH tipo inatento; TDAH/TC= TDAH tipo combinado; BRIEF= Behavior Rating Inventory of Executive Functions. ${ }^{a}$ Tests post-hoc de Tuckey (igualdad de varianzas) y de Games-Howell (no igualdad de varianzas). IRC= Índice de Regulación Comportamental; IM= Índice de Metacognición. CEG= Composición Ejecutiva Global. * Todas las $F$ son estadísticamente significativas $(p<0,001)$.

En relación con la segunda hipótesis (el grupo con TDAH de tipo inatento mostrará alteraciones en la capacidad de metacognición y el grupo con TDAH de tipo combinado lo hará tanto en la capacidad de metacognición como en la de regulación comportamental) se observaron diferencias significativas entre ambos grupos con TDAH. Las diferencias entre estos dos grupos se dieron en las dimensiones de Inhibición y Control Emocional (incluidas en el Índice de Regulación Comportamental) y en la de Control (incluida en el Índice de Metacognición) así como en el propio Índice de Regulación Comportamental (IRC) y en la Composición Ejecutiva Global (CEG). El grupo que obtuvo puntuaciones más elevadas, es decir, que se mostró más afectado en las dimensiones correspondientes fue, en todas las ocasiones, el grupo con TDAH de tipo combinado (ver Tabla 1).

Con respecto a la tercera hipótesis (las variables ecológicas que evalúan las disfunciones en la capacidad de metacognición y en el con- 
Tabla 2. Coeficientes de correlación parcial entre las dimensiones de la escala BRIEF y la sintomatología de TDAH. (Controlando la edad) $(N=58)$

\begin{tabular}{lcc}
\hline \multicolumn{1}{c}{ Dimensiones de la escala BRIEF } & Síntomas de Inatención $^{\mathrm{a}}$ & Síntomas de hiperactividad/impulsividad $^{\mathrm{a}}$ \\
\hline Cambio & 0,65 & 0,49 \\
Inhibición & 0,74 & 0,79 \\
Control Emocional & 0,60 & 0,65 \\
IRC & 0,73 & 0,71 \\
Iniciativa & 0,85 & 0,50 \\
Memoria de Trabajo & 0,88 & 0,54 \\
Planificación-Organización & 0,78 & 0,50 \\
Organización de Materiales & 0,68 & 0,52 \\
Control & 0,82 & 0,68 \\
IM & 0,86 & 0,59 \\
CEG & 0,86 & 0,67 \\
\hline
\end{tabular}

Nota. Todas las correlaciones son significativas $(\mathrm{p}<0,01)$; BRIEF $=$ Behavior Rating Inventory of Executive Functions; ${ }^{\mathrm{a}}$ Síntomas de inatención y síntomas de hiperactividad-impulsividad evaluados según la escala DSM-IV-TR. IRC= Índice de Regulación Comportamental; IM= Índice de Metacognición; $\mathrm{CEG}=$ Composición Ejecutiva Global.

trol del comportamiento estarán relacionadas con la sintomatología de inatención y de hiperactividad/impulsividad, respectivamente, del TDAH), los análisis correlacionales arrojan los siguientes resultados (véase la Tabla 2): la sintomatología de hiperactividad-impulsividad correlaciona de manera positiva con todas las dimensiones evaluadas en la escala BRIEF. Las correlaciones más elevadas (por encima de 0,60$)$ se dan con la Inhibición $(r=0,79)$, el Control Emocional $(r=0,65)$, el Control $(r=$ 0,68 ) y el IRC (Índice de Regulación Comportamental) $(r=0,71)$ asi como en la CEG $(r$ $=0,67)$. Al igual que la sintomatología de hiperactividad/impulsividad, los síntomas de inatención correlacionaban con todas las dimensiones de la escala BRIEF; en este caso, la mejor parte de las correlaciones eran superiores a 0,65 .

\section{DISCUSIÓN}

Siguiendo planteamientos paralelos a los de otras investigaciones (Armstrong, 2001; Chhabildas et al., 2001), los índices de metacognición (IM) y de regulación comportamental (IRC) de la escala BRIEF podrían equipararse a lo que en psicología cognitiva se define como conocimiento procedimental y conocimiento declarativo (Gagné, 1991), es decir, a los aspectos comportamentales y cognitivos respectivamente o, según una reciente teoría, a los sistemas "hot" y "cool" que plantean la existencia de dos perfiles distintos dentro del funcionamiento ejecutivo (Castellanos, Sonuga-Barke, Milham y Tannock, 2006; Geurts, Van Der Oord y Crone, 2006; Zelazo, Qu y Müller, 2005). Los resultados de nuestro estudio muestran diferencias significativas en el funcionamiento ejecutivo entre el grupo de control y los grupos con TDAH, tanto en los aspectos comportamentales (IRC) como en los cognitivos (IM). En la consideración del funcionamiento ejecutivo de los tipos inatento y combinado observamos diferencias en el Índice de Regulación Comportamental y en dos de sus dimensiones (Inhibición y Control Emocional). Por tanto, según los resultados, el grupo con TDAH de tipo combinado manifestó mayores dificultades que el tipo inatento para resistir a los impulsos así como una mayor labilidad emocional y repentinos cambios de humor. La ausencia de diferencias significativas en la dimensión Cambio da cuenta de que ambos tipos de TDAH mostraron dificultades similares, con respecto al grupo de control, para alternar de una actividad a otra o 
de un aspecto del problema a otro cuando las circunstancias así lo requerían, es decir, mostraron alteraciones en flexibilidad cognitiva. En lo que respecta al índice de metacognición (Iniciativa, Memoria de Trabajo, Organización y Planificación, Organización de Materiales y Control) no podemos suponer diferencias generalizadas entre los tipos inatento y combinado, ya que solamente hemos encontrado desigualdad significativa en una de las cinco escalas correspondientes a este Índice, concretamente en la de Control.

De esta forma, aunque no se evidencia una distinción generalizada entre los tipos con respecto a este índice, si podemos afirmar que el grupo con TDAH de tipo combinado manifiesta, con respecto al tipo inatento, una menor conciencia de su propio comportamiento y del impacto que este comportamiento puede tener en sus interacciones sociales; esta afectación en la capacidad para el control del comportamiento puede llevarle a tener dificultades para desarrollar una determinada tarea o para adquirir hábitos de trabajo. Chhabildas et al. (2001) se sitúan en una posición similar afirmando que ambos tipos pueden diferenciarse sólo en los aspectos comportamentales de la inhibición pero no en los cognitivos. La ausencia de diferencias significativas entre los tipos inatento y combinado del TDAH en las dimensiones de Cambio, Iniciativa, Planificación-Organización, Memoria de Trabajo y Organización de Materiales además de en el IM, da cuenta de que ambos tipos muestran dificultades similares, con respecto al grupo de control, para: (a) alternar de un aspecto de un problema a otro o de una actividad a otra cuando las circunstancias lo demandan, es decir, alteraciones en flexibilidad cognitiva (Loeber, Brinthaupt y Green, 1990; Loeber, Green y Lahey, 1990), (b) iniciar una determinada actividad sin ser inducido a ello, (c) planificar, organizar, controlar sus propias acciones, (d) mantener en la memoria de trabajo dicha actividad y (e) ordenar y organizar los materiales de clase o de juego. Las diferencias de ambos tipos en el Índice de Metacognición con respecto al grupo de control suponen una dificultad similar para la resolución activa de problemas en diferentes contextos lo cual lleva a considerar disfunciones en la inteligencia fluida.
A continuación, nos centraremos en los resultados obtenidos en las dimensiones de Inhibición y Memoria de Trabajo que, como hemos mencionado, aseguran, según algunos estudios, la diferenciación entre tipos (Gioia et al., 2000). El análisis de varianza confirma que la capacidad de inhibición (dimensión homónima de la escala BRIEF) está significativamente más alterada en el grupo con TDAH de tipo combinado que en grupo de tipo inatento. En cambio, la memoria de trabajo (según la dimensión análoga de la escala BRIEF) está afectada por igual en ambos tipos de TDAH con respecto al grupo de control. Según Isquith y Gioia (2000) y Gioia et al. (2000) el control inhibitorio facilita la auto-regulación y la memoria de trabajo la atención. Por tanto, nuestros resultados ponen de manifiesto una menor capacidad de autorregulación en el caso del tipo combinado pero una alteración similar en la capacidad de atención en ambos tipos.

Con respecto a la relación entre la sintomatología del TDAH y las disfunciones ejecutivas, la principal conclusión es que los síntomas de hiperactividad-impulsividad correlacionan de forma más débil con dichas disfunciones que los de inatención. La Composición Ejecutiva Global (CEG) muestra una relación lineal más fuerte con los síntomas de inatención que con los de hiperactividad/impulsividad lo que confirma que son estos síntomas los que están altamente relacionados con las disfunciones ejecutivas presentes en los niños con TDAH. Los síntomas de hiperactividad/impulsividad sí muestran una relación similar a la de la sintomatología inatencional con el IRC, lo que confirma los resultados del análisis diferencial en los que el tipo combinado y, por tanto con más síntomas de hiperactividad-impulsividad, se encontraba más afectado que el tipo inatento en este índice. En las dimensiones pertenecientes a cada uno de los índices (metacognición y control comportamental) los resultados son ambiguos. En contra de lo que cabría esperar, las dimensiones de Inhibición, Cambio y Control Emocional (incluidas en el IRC) manifiestan una relación similar con los síntomas de inatención que con los de hiperactividad/impulsividad. Las dimensiones correspondientes al Índice de Metacognición se comportan en con- 
sonancia a su índice de referencia, es decir, tienen una relación más potente con la sintomatología de inatención que con la de hiperactividad/impulsividad.

Nuestro objetivo ahora es dilucidar por qué la sintomatología inatencional está altamente relacionada con todas las dimensiones de la escala BRIEF mientras que los síntomas de hiperactividad-impulsividad establecen una relación más moderada. Sabemos que la capacidad atencional tiene unas manifestaciones cognitivas y unas manifestaciones comportamentales (Armstrong, 2001). Esto puede explicar por qué los síntomas de inatención mantienen estos índices de correlación, tanto con el IM como con el IRC, es decir, tanto con las manifestaciones cognitivas como con las comportamentales del funcionamiento ejecutivo. Podríamos concluir que, desde el punto de vista del profesor, tanto el Índice de Metacognición como el de Control de Comportamiento están relacionados de manera potente con la sintomatología de inatención y, en menor medida, con la de hiperactividadimpulsividad. Según los resultados, el Índice de Metacognición, es decir, la habilidad para iniciar, planificar, organizar y mantener en la memoria de trabajo (Isquith y Gioia, 2000) los pasos necesarios para llegar a una meta propuesta correlaciona de manera más elevada con la sintomatología inatencional que con la de hiperactividad/impulsividad, mientras que el Índice de Regulación Comportamental, y sus dimensiones, es decir, la resolución de problemas de forma metacognitiva, teniendo conciencia del control de la conducta necesario para llegar a la solución final, mantiene una relación similar con ambos tipos de síntomas, de inatención y de hiperactividad/impulsividad.

Según los resultados, podemos ofrecer las siguientes conclusiones. En primer lugar, los grupos con TDAH manifiestan disfunciones ejecutivas, desde el punto de vista ecológico, con respecto al grupo de control. La segunda conclusión importante es que los tipos inatento y combinado del TDAH se diferencian de manera general en los aspectos comportamentales del funcionamiento ejecutivo y de forma específica en los aspectos cognitivos. En tercer lugar, podemos decir que, con respecto a la capacidad de metacognición y el control del comportamiento del funcionamiento ejecutivo, son los síntomas de inatención los que están relacionados de manera más potente. Por último, esperamos que la consideración de estos perfiles en el funcionamiento ejecutivo determine la puesta en marcha de programas de tratamiento más concretos.

\section{REFERENCIAS}

American Psychiatric Association (2000). Diagnostic and statistical manual of mental disorders ( $4^{\mathrm{a}} \mathrm{ed}$. Texto revisado). Washington, DC: Author.

Armstrong, T. (2001). Síndrome de déficit de atención con o sin hiperactividad (ADD/ADHD). Estrategias en el aula. Barcelona: Paidós.

Barkley, R. (1997). Behavioral inhibition, sustained attention, and executive functions: constructing a unifying theory of ADHD. Psychological Bulletin, 121, 65-94.

Barkley, R. (2006). Attention deficit hyperactivity disorder: a handbook for diagnosis and treatment ( $3^{\mathrm{a}} \mathrm{ed}$.). New York: Guilford Press.

Barkley, R., Edwards, G., Laneri, M., Fletcher, K., y Metevia, L. (2001). Executive functioning, temporal discounting, and sense of time in adolescents with attention deficit hyperactivity disorder (ADHD) and oppositional defiant disorder (ODD). Journal of $A b$ normal Child Psychology, 29, 541-546.

Barkley, R., Grodzinsky, G., y DuPaul, G.J. (1992). Frontal lobe functions in attention deficit disorder with and without hyperactivity: A review and research report. Journal of Abnormal Child Psychology, 20, 163-188.

Barkley, R., y Murphy, K. (1998). Attention-deficit hyperactivity disorder: A clinical workbook ( $2^{\mathrm{a}}$ ed.). New York: Guilford Press.

Berlin, L., Bohlin, G., Nyberg, L., y Janols, L.O. (2004). How well do measures of inhibition and other executive functions discriminate between children with ADHD and controls? Child Neuropsychology, 10, 1-13.

Brito, G. (1987). The Conners Abbreviated teacher rating scale: developmental of norms in Brazil. Journal of Abnormal Child Psychology, 15, 511-518.

Castellanos, F.X., Sonuga-Barke, E., Milham, M., y Tannock, R. (2006). Characterizing cognition in ADHD: beyond executive dysfunction. Trends in Cognitive Sciences, 10, 117-123.

Chhabildas, N., Pennington, B.F., y Willcutt, E.G. (2001). A comparison of the Neuropsychological profiles of the DSM-IV Subtypes of ADHD. Journal of Abnormal Child Psychology, 29, 529-540.

Conners, C. (1969). A teacher rating scale for use in drug study with children. American Journal of Psychiatry, 126, 884-888. 
Conners, C., Sitarenios, G., Parker, J., y Epstein, J. (1998). Revision and restandarization of the Conners scale teacher rating (CTRS-R): factor structure, reliability and criterion validity. Journal of Abnormal Child Psychology, 26, 279-291.

Crystal, D.S., Ostrander, R., Chen, R., y August, G.J. (2001). Multimethod Assessment of Psychopathology among DSM-IV subtypes of children with attentiondeficit/hyperactivity disorder: self-, parent, and teacher reports. Journal of Abnormal Child Psychology, 29, 189-205.

DeBonis, D.A., Ylvisaker, M., y Kundert, D.K. (2000). The relationship between ADHD theory and practice: a preliminary investigation. Journal of Attention Disorders, 4, 161-173.

DuPaul, G.J., Anastopoulos, A.D., McGoey, K.E., Power, T.J., Reid, R., e Ikeda, M.J. (1998). Teacher ratings of attention deficit hyperactivity disorder symptoms: Factor structure and normative data. Psychological Assessment, 9, 436-444.

Ferro, R., Vives, M.C., y Ascanio, L. (2010). Aplicación de la terapia de interacción padres-hijos en un caso de trastorno negativista desafiante. Revista de Psicopatología y Psicología Clínica, 15, 205-214.

Fonseca-Pedrero, E., Paino, M., Lemos-Giráldez, S., y Muñiz, J. (2011). Prevalencia de la sintomatología emocional y comportamental en adolescentes españoles a través del Strengths and Difficulties Questionnaire (SDQ). Revista de Psicopatología y Psicología Clínica, 16, 17-25.

Frías, A., Carrasco, A., Fernández, M.J., García, A., y García, B. (2009). Comorbilidad entre trastornos de conducta y depresión mayor en la adolescencia: Prevalencia, teorías explicativas y estatus nosológico. Revista de Psicopatología y Psicología Clínica, 14, 1-16.

Gagné, E.D. (1991). La psicología cognitiva del aprendizaje escolar. Madrid: Visor.

Games, P.A., Keselman, H.J., y Rogan, J.C. (1981). Simultaneous pairwise multiple comparison procedures for means when sample sizes are unequal. Psychological Bulletin, 90, 594-598.

Geurts, H.M., Van der Oord, S., y Crone, E. (2006). Hot and cool aspects of cognitive control in children with ADHD: decision-making and inhibition. Journal of Abnormal Child Psychology, 34, 813-824.

Geurts, H.M., Verté, S., Oosterlaan, J., Roeyers, H., y Sergeant, J.A. (2004). How specific are executive functioning deficits in attention deficit hyperactivity disorder and autism? Journal of Child and Psychology Psychiatry, 45, 836-854.

Gioia, G.A., Isquith, P.K., Guy, S.C., y Kenworthy, L. (2000). Test Review Behavior Rating Inventory of Executive Function. Child Neuropsychology, 6, 235-238.

Gomez, R., Burns, G.L., Walsh, J.A., y Alves De Moura, M. (2003). A multitrait-multisource confirmatory fac- tor analytic approach to the construct validity of ADHD rating scales. Psychological Assessment, 15, 3-16.

Gomez, R., Harvey, J., Quick, C., Scharer, I., y Harris, G. (1999). DSM-IV AD/HD: confirmatory factor models, prevalence, and gender and age differences based on parent and teacher ratings of Australian primary school children. Journal of Child Psychology and Psychiatry, 40, 265-274.

Goyette, C.H., Conners, C.K., y Ulrich, R.F. (1978). Normative data on revised Conners parent and teacher rating scales. Journal of Abnormal Child Psychology, 6, 221-236.

Hartman, C.A., Willcutt, E.G., Rhee, S.H., y Pennington, B.F. (2004). The relation between Sluggish Cognitive Tempo and DSM-IV ADHD. Journal of Abnormal Child Psychology, 32, 491-503.

Hinshaw, S.P., Carte, E.T., Fan, C., Jassy, J.S., y Owens, E. B. (2007). Neuropsychological functioning of girls with attention-deficit/hyperactivity disorder followed prospectively into adolescence: evidence for continuing deficits? Neuropsychology, 21, 263-273.

Houghton, S., Douglas, G., West, J., Whiting, K., Wall, M., Langsford, S., Powell, L., y Carroll, A. (1999). Differential patterns of executive function in children with attention-deficit hyperactivity disorder according to gender and subtype. Journal of Child Neurology, 14, 801-805.

Hoza, B., Gerdes, A.C., Hinshaw, S.P., Arnold, L.E., Pelham Jr., W.E., Molina, B.S., Abikoff, H.B., Epstein, J.N., Greenhill, L.L., Hechtman, L., Odbert, C., Swanson, J.M., y Wigal, T. (2004). Self-perceptions of competence in children with ADHD and comparison children. Journal of Consultimg and Clinical Psychology, 72, 382-391.

Hoza, B., Pelham, W.E., Waschbusch, D.A., Kipp, H., y Owens, J.S. (2001). Academic task persistence of normally achieving ADHD and control boys: performance, self-evaluations, and attributions. Journal of Consultimg and Clinical Psychology, 69, 271-283.

Hughes, C., y Graham, A. (2002). Measuring executive functions in childhood: Problems and Solutions? Child and Adolescent Mental Health, 7, 131-142.

Isquith, P.K., y Gioia, G.A. (2000). Brief predictions of ADHD: clinical utility of the Behavior Rating Inventory of Executive Function for detecting ADHD subtypes in children. Archives of Clinical Neuropsychology, 15, 653-850.

Kazdin, A.E., Esveldt-Dawson, K., y Loar, L.L. (1983). Correspondence of teacher ratings and direct observations of classroom behavior of psychiatric impatient children. Journal of Abnormal Child Psychology, 11, 549-564.

Lahey, B.B., Pelham, W.E., Stein, M.A., Loney, J., Trapani, C., Nugent, K., Kipp, H., Schmidt, E., Lee, S., Cale, M., Gold, E., Hartung, C.M., Willcutt, E., y Baumann, 
B. (1998). Validity of DSM-IV attention-deficit/hyperactivity disorder for young children. Journal of American Academy of Child and Adolescent Psychiatry, 37, 695-702.

Loeber, R., Brinthaupt, V.P., y Green, S.M. (1990). Attention deficits, impulsivity, and hyperactivity with or without conduct problems: relationships to delinquency and unique contextual factors. En R.J. McMahon y R. de V. Peters (Eds.), Behavior Disorders of Adolescence (pp.39-61). New York: Plenum Press.

Loeber, R., Green, S.M., y Lahey, B.B. (1990). Mental Health professionals' perception of the utility of children, mothers, and teachers as informants on childhood psychopathology. Journal of Clinical Child Psychology, 19, 136-143.

Maganto, C. (1995). Instrumentos aplicados para la evaluación y el diagnóstico en psicopatología infantil. En J. Rodríguez (Ed.), Psicopatología del niño y del adolescente (pp.191-282). Sevilla: Manuales Universitarios.

Martínez Arias, R. (1995). Metodología de las Ciencias del Comportamiento. Psicometría: Teoría de los tests psicológicos y educativos. Madrid: Síntesis Psicología.

Miyake, A., Friedman, N.P., Emerson, M.J., Witzki, A.H., y Howerter, A. (2000). The unity and diversity of executive functions and their contributions to complex frontal lobe tasks: A latent variable analysis. Cognition and Psychology, 41, 49-100.

Nigg, J.T. (2006). What causes ADHD? Understanding what goes wrong and why. New York: Guilford Press.

Nigg, J.T., Blaskey, L., Huang-Pollock, C., y Rappley, M.D. (2002). Neuropsychological executive functions and ADHD DSM-IV subtypes. Journal of the American Academy of Child and Adolescent Psychiatry, 41, 5966.

Nigg, J.T., Blaskey, L., Stawicki, J., y Sachek, J. (2004). Evaluating the endophenotype model of ADHD neuropsychological deficit: results for parents and siblings of children with DSM-IV ADHD combined and inattentive subtypes. Journal of Abnormal Child Psycho$\log y, 113,614-625$.

Nigg, J.T., Stavro, G., Ettenhofer, M., Hambrick, D., Miller, T., y Henderson, J.M. (2005). Executive functions and ADHD in adults: Evidence for selective effects on ADHD symptom domains. Journal of Abnormal Psychology, 114, 706-717.

Orjales Villar, I. (2002). Déficit de atención con hiperactividad. Manual para padres y educadores. Madrid: CEPE.

Ozonoff, S. (2000). Componentes de la función ejecutiva en el autismo y otros trastornos. En J. Russell (Ed.), El autismo como trastorno de la función ejecutiva (pp. 177-201). Madrid: Médica Panamericana.
Pelechano, V. (2007). Viejas y nuevas cuestiones en las viejas y nuevas terapias psicológicas. Revista de Psicopatología y Psicología Clínica, 12, 71-90.

Pennington, B.F., y Ozonoff, S. (1996). Executive functions and developmental psychopathology. Journal of Child Psychology and Psychiatry and Allied Disciplines, 37, 51-87.

Robbins, T.W., James, M., Owen, A.M., Sahakian, B.J., Lawrence, A.D., y McIness, L. (1998). A study of performance on tests from the CANTAB battery sensitive to frontal lobe dysfunction in a large sample of normal volunteers: Implications for theories of executive functioning and cognitive aging. Journal of the International Neuropsychological Society, 4, 474-480.

Rowe, K.S., y Rowe, K.J. (1997). Norms for parental ratings on Conners' abbreviated Parent-Teacher-Questionnaire: implications for the design of behavioral rating inventories and analyses of data derived from them. Journal of Abnormal Child Psychology, 25, 425-451.

Scheres, A., Oosterlaan, J., Geurts, H., Morein-Zamir, S., Meiran, N., Schut, H., Vlasveld, L., y Sergeant, J.A. (2004). Executive functioning in boys with ADHD: primarily an inhibition deficit? Archives of Clinical Neuropsychology, 19, 569-594.

Sergeant, J.A., Geurts, H., Huijbregts, S., Scheres, A., y Oosterlaan, J. (2003). The top and bottom of ADHD: A neuropsychological perspective. Neuroscience Biobehavioral Review, 27, 583-592.

Shulman, L. (1986). Those who understand: Knowledge growth in teaching. Educational Research, 15, 4-14.

Valero, L. (1997). La evaluación del comportamiento infantil: características y procedimientos. Apuntes de Psicología, 51, 51-79.

Weyandt, L.L., Rice, J.A., Linterman, I., Mitzlaff, L., y Emert, E. (1998). Neuropsychological performance of a sample of adults with ADHD, developmental reading disorder, and controls. Devevelopmental Neuropsychology, 14, 643-656.

Willcutt, E.G., Chhabildas, N., y Pennington, B.F. (2001). Validity of DSM-IV subtypes of ADHD. The ADHD Report, 9, 2-5.

Willcutt, E.G., Pennington, B.F., Chhabildas, N.A., Olson, R.K., y Hulslander, J.L. (2005). Neuropsychological analyses of comorbidity between reading disorder and ADHD: in search of the common deficit. Developmental Neuropsychology, 27, 35-78.

Zelazo, P. D., Qu, L., y Müller, U. (2005). Hot and cool aspects of executive function: Relations in early development. En W. Schneider, R. Schumann-Hengsteler, y B. Sodian (Eds.), Young children's cognitive development: Interrelationships among executive functioning, working memory, verbal ability, and theory of mind (pp. 71-93). Mahwah, NJ: Erlbaum. 